\title{
Bedside Teaching: The Meeting-to-Meeting Model
}

\author{
Mohammed Garout', Abdulelah Nuqali², Ahmad Alhazmi' ${ }^{3}$, Hani Almoallim ${ }^{4}$ \\ ${ }^{1}$ Department of Community Medicine and Pilgrims Healthcare, Umm Al-Qura University, Makkah, Saudi Arabia \\ ${ }^{2}$ Department of Medicine, The George Washington University, Washington DC, USA \\ ${ }^{3}$ Department of Surgery, Umm Al-Qura University, Makkah, Saudi Arabia \\ ${ }^{4}$ Department of Internal Medicine, Umm Al-Qura University, Makkah, Saudi Arabia \\ Email: hanialmoallim@gmail.com, Abdulelah.n@gmail.com
}

Received 11 June 2016; accepted 17 July 2016; published 21 July 2016

Copyright @ 2016 by authors and Scientific Research Publishing Inc.

This work is licensed under the Creative Commons Attribution International License (CC BY).

http://creativecommons.org/licenses/by/4.0/

(c) (i) Open Access

\section{Abstract}

The Meeting-to-Meeting Model is designed to provide a medical educator with an easy-to-use guide to conduct efficient bedside teaching sessions in inpatient settings. Here, we summarize some of the strategies described in the literature, which we have modified in order to make the model more practical. The model comprises five main steps: Meeting I, corridor I, patient encounter, corridor II, and meeting II. We also briefly describe adult learning theory in relation to BST. Further research is needed to assess the generalizability of the model and to evaluate its effect on educational outcomes.

\section{Keywords}

Bedside Teaching, Clinical Skills, Medical Education, Medical Student, Clinical Competence, Teaching Methods

\section{Introduction}

Many bedside teaching (BST) models have been published. Stichrath et al. repackaged the high-yield teaching activities during BST to create the MiPLAN model (Stickrath et al., 2013). "M" represents meeting, "I" represents the 5 i's (introduction, in the moment, inspection, interruption, and independent thoughts), and PLAN prioritizes the teaching opportunities (patient care, learners' questions, attending physician's agenda, and next steps). Dent, on the other hand, focused on tutor allocation and the student's behavior during patient encounters (Dent \& Harden, 2013). Ramani et al., Gonzalo, and Spencer also discussed various aspects of BST (Ramani et al., 2003; Gonzalo et al., 2013; Spencer, 2003). 
The Meeting-to-Meeting Model summarizes some of the strategies that have been described in the literature. Some modifications have been added, however, in order to make the model more practical.

The model comprises five main steps: Meeting I, corridor I, patient encounter, corridor II, and meeting II. It can be applied to more than one patient during an individual BST session, as shown in Figure 1 and Table 1. Further research needs to be done to assess the model's generalizability and to evaluate its effect on educational outcomes.

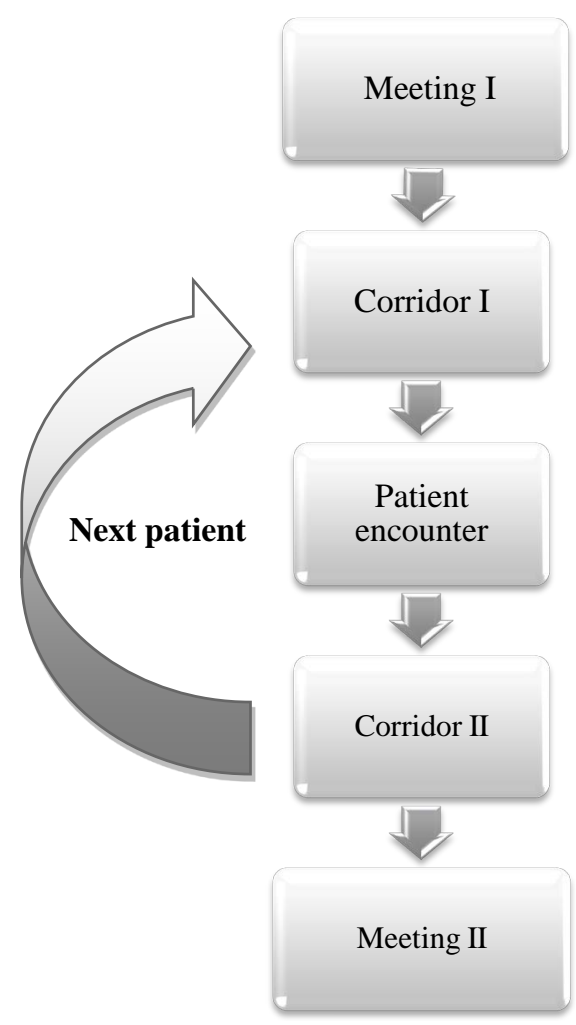

Figure 1. Meeting-to-meeting model.

Table 1. Steps and components of the meeting-to-meeting model.

\begin{tabular}{cc}
\hline Step & Components \\
\hline Meeting I & Introduction \\
& Set the agenda \\
& Tutors' preparation \\
& Students' preparation \\
Corridor I & Enforce safety standards \\
& Preparation \\
& Ensure that everyone knows their task \\
& Communication \\
Patient encounter & Role allocation \\
& Case presentation \\
& Clinical examination \\
& Engage everyone \\
Corridor II & Involve patients \\
& Debriefing \\
& Reflection \\
Meeting II & Discussion and answering questions \\
& Students' feedback \\
\end{tabular}




\section{Steps and Components of the Meeting-to-Meeting Model}

\subsection{Meeting I}

\subsubsection{Introduction}

A decent start to a meeting is the first step toward developing good communication skills between the tutor and the students. Tutors should try to get to know the students, make them feel free to learn or ask questions, and provide a good educational environment. This approach will be positively reflected in the quality of BST and will make it more effective.

\subsubsection{Set the Agenda}

Determining the objectives and setting up the goals of each session will save time and will organize the students as well as the tutor. In addition, this step helps the students achieve the maximum benefits. Tutors should set the agenda for the educational activities for each session and what they plan to discuss. They should emphasize the following:

- Teamwork and collaboration

- Instructions for effective case presentation

- Encouragement of learning and discussion

- Determination of the cases and topics to be covered

- Skills that should be mastered

- Division of tasks among the students

- Description of how this session will be conducted

\subsubsection{Tutors' Preparation}

Tutors should be prepared for BST according to the strategies mentioned in the literature; that is, the sessions should be trainee-specific, disease-specific, and patient-specific, and the tutor should be mentally prepared.

\subsubsection{Students' Preparation}

Both tutors and students have a major role in ensuring the productivity and effectiveness of BST. Students could suggest learning objectives, which allow the tutor to tailor educational activities to meet their needs. They should also do the following:

- Prepare the cases before starting BST

- Read about the topics

- Try to note the physical findings

- Develop communication skills with patients and relatives to allow them to be more cooperative with the team

- Discuss the case with their colleagues to enhance the value of teamwork

- Be responsible and independent learners

- Interact with the tutor effectively to improve their professional attitude

- Establish a learning contract to enhance their commitment

\subsection{Corridor I}

\subsubsection{Enforce Safety Standards}

Safety comes first. Tutors should inform and teach the students about safety and standard health precautions. They should remind their students to wash their hands before and after physical examinations, which will minimize cross-contamination and transmission of infection.

\subsubsection{Preparation}

Tutors should prepare students for possible diagnostic or therapeutic procedures before patient encounters. Some patients may need to have their dressing changed, e.g., patients with bed sores. It is useful to prepare the dressing trolley before encountering a patient. The tutor could give the students a brief description of how to do a proper dressing, and then give them a chance to do it themselves under direct supervision. Moreover, students should prepare the patient's file and any recent laboratory investigations or recent imaging studies, which are valuable materials to discuss and interpret. 


\subsubsection{Ensure That Everyone Knows Their Task}

Tutors should remind students about their tasks, describe these tasks, and then make sure students understand their roles, as this procedure will help tutors run the session smoothly.

\subsection{Patient Encounter}

\subsubsection{Communication}

Communication skills are an art and if the students master them, patients will be more cooperative, which will maximize students' opportunities to be exposed to more cases. Always ensure proper communication with the patients and describe what the plan is. Show them proper care and listen to their concerns. Good communication skills will build a trust bridge with the patients.

It is the patient's right to know who will be doing the examination and why. Tutors should introduce themselves and their students after receiving permission from the patient in order to ensure his or her privacy. Always remember that patients are human beings, not educational objects. Therefore, they must be fully respected. Remember to cover patients after finishing a clinical examination and to respect their desires if they want to stop the examination if they are uncomfortable or in pain.

\subsubsection{Role Allocation}

As the team reaches a patient preceding an encounter, the distribution of the tutor and students around the patient's bed is an important issue to be discussed. Three possibilities for role allocation are mentioned in A Practical Guide for Medical Teachers (Dent \& Harden, 2013):

- Demonstrator model: The tutor stands to the right side of the patient and demonstrates how to do history taking and a physical examination while the students are distributed around the patient and simply observe.

- Tutor model: The tutor stands to the left side of the patient and observes each student in turn during history taking and physical examination and then gives comments during the observation.

- Observer model: The tutor stands away from the patient and the students in turn during history taking and the physical examination. At the end, the tutor provides feedback and discusses the findings.

\subsubsection{Case Presentation}

The best location for the case presentation is debatable. Some learners prefer to present their cases in a conference room for several reasons:

1) Freedom to discuss: "to explore academic aspects of the disease or tangents not immediately applicable to the patient involved" (7)

2) Concern for patients' feelings

3) Comfort, privacy, access to a blackboard and X-rays

On the other hand, a study showed that most patients (85\%) preferred their cases to be presented in front of them, even though some of them did not fully understand the discussion (Wang-Cheng et al., 1989).

A combination of a conference room and the patient's bedside in a case presentation is an acceptable approach and is preferred by about $53 \%$ of the learners (Nair et al., 1997).

Finally, a corridor is not an ideal venue for a case presentation for either party: Patients think it may break their confidentiality and, as the study by Nair et al. showed, only $2 \%$ of learners preferred hospital corridors (Nair et al., 1997).

\subsubsection{Clinical Examination}

It is important for the students to be familiar with normal and then abnormal physical findings in a patient. The magical mix that could enhance the physician-patient relationship is as follows: obtaining permission before an examination, being kind and polite to the patient, explaining every step or examination prior to performing it, staying away from areas of pain, and covering the patient after the examination.

\subsubsection{Engage Everyone}

Tutors should encourage students to actively participate in the BST session. This approach will make the session more interactive and interesting. 


\subsubsection{Involve Patients}

In order to make BST successful, patients and their families are encouraged to contribute to the details of the history of the illness, which gives them a chance to ask questions, to understand what is being done and why, and to know what the management plan is. Finally, efforts must be made to simplify and explain the information by using easy and understandable language.

\subsection{Corridor II}

\subsubsection{Debriefing}

Having approached this step of BST, the tutor has to summarize everything he or she has done so far, including important points of history, patient concerns, clinical examination findings, and laboratory results or abnormal results found on imaging studies. This summary can make the case clearer and highlight the important points that need to be discussed later.

\subsubsection{Reflection}

To improve the student's medical knowledge and avoid repeating mistakes, tutors should request that students evaluate their performance and highlight their strengths as well as areas for improvement in their BST skills.

\subsubsection{Discussion and Answering Questions}

Tutors should actively work on improving students' analytical and clinical reasoning skills and discuss all aspects of a case, e.g., by making a list of possible differential diagnoses and building an appropriate management plan. Furthermore, the tutor might give students the chance to run the discussion by themselves. Tutors should answer the students' questions, clarify what they need to understand, and highlight important points that are needed at their level.

\subsection{Meeting II}

\subsubsection{Students' Feedback}

When the tutor gives the students time to reflect, he or she should also receive their feedback on the way BST was conducted and whether they liked it or not, as well as whether they have any suggestions on how they might prefer the next session to be run. Feedback will help the tutor improve the quality of teaching and help the learners develop a formal attitude to feedback.

\subsubsection{Plan}

At the end of the session, the tutor should plan for the next one and involve the students in setting up the objectives, e.g., what they need to learn, which subject they need to discuss, or which skills they need to practice. In addition, the tutor should request that the students think about and prepare specific tasks for discussion during the next session.

\section{Adult Learning and BST}

Tutors should be aware of the constructivist learning theory and adult learning principles, as understanding and using these theories will help clinical educators to be more effective during BST (Stickrath et al., 2013; Spencer, 2003). Learners come to the BST session with pre-existing knowledge from past experiences and will be ready to receive new knowledge and information from their tutors. After that, motivated students will look for additional information to help them improve their understanding.

Therefore, applying these concepts during BST will effectively help students to become competent and broadthinking learners. This learning process has three main steps, as shown in Figure 2: 1) Pre-existing knowledge: It is essential that tutors or clinical educators assess past experiences and information and then identify them by activating the students through brainstorming and briefings. In addition, tutors should take into consideration any relevant knowledge that helps with constructing and building new knowledge. 2) New knowledge: Tutors should help learners to use and understand new knowledge and then provide a bridge between pre-existing knowledge and new information. This will help students construct a logical framework that can accommodate new information. 3) Independent, self-directed learning: Clinical educators should help learners develop metacog- 


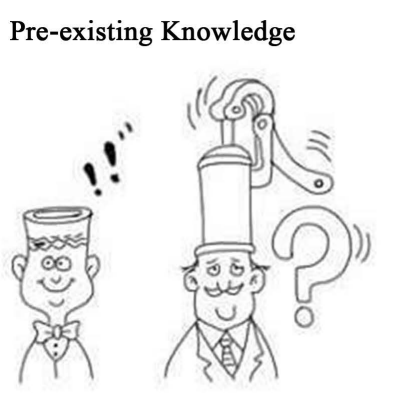

1

Tutor helps the student to identify pre-existing knowledge.

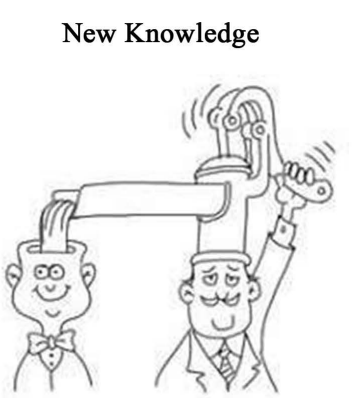

2

Tutor helps the student to understand \& utilize new knowledge.
Independent;

Self-directed

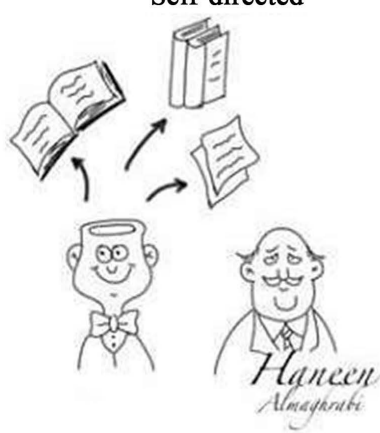

3

Tutor helps the student to become self-directed learners.

Figure 2. The constructivist learning theory.

nitive skills, as well as monitor their thinking during and after the session. This will help students to use and add new information in a way that allows them to apply it in different situations.

\section{Acknowledgements}

The authors thank Alzaidi Chair of Research in Rheumatic Diseases at Umm Alqura University for supporting and supervising this paper. We also thank Dr. Allison Vanderbilt for her helpful comments on the manuscript and Dr. Haneen Almaghrabi for her drawing of the constructivist learning theory.

\section{References}

Dent, J., \& Harden, R. M. (2013). A Practical Guide for Medical Teachers. London: Churchill Livingstone.

Gonzalo, J. D., Heist, B. S., Duffy, B. L., Dyrbye, L., Fagan, M. J., Ferenchick, G. et al. (2013). The Art of Bedside Rounds: A Multi-Center Qualitative Study of Strategies Used by Experienced Bedside Teachers. Journal of General Internal Medicine, 28, 412-420. http://dx.doi.org/10.1007/s11606-012-2259-2

Nair, B. R., Coughlan, J. L., \& Hensley, M. J. (1997). Student and Patient Perspectives on Bedside Teaching. Medical Education, 31, 341-346. http://dx.doi.org/10.1046/j.1365-2923.1997.00673.x

Ramani, S., Orlander, J. D., Strunin, L., \& Barber, T. W. (2003 Whither Bedside Teaching? A Focus-Group Study of Clinical Teachers. Academic Medicine: Journal of the Association of American Medical Colleges, 78, 384-390. http://dx.doi.org/10.1097/00001888-200304000-00014

Spencer, J. (2003). Learning and Teaching in the Clinical Environment. BMJ (Clinical Research ed), 326, 591-594. http://dx.doi.org/10.1136/bmj.326.7389.591

Stickrath, C., Aagaard, E., \& Anderson, M. (2013). MiPLAN: A Learner-Centered Model for Bedside Teaching in Today’s Academic Medical Centers. Academic Medicine: Journal of the Association of American Medical Colleges, 88, 322-327. http://dx.doi.org/10.1097/ACM.0b013e318280d8f7

Wang-Cheng, R. M., Barnas, G. P., Sigmann, P., Riendl, P. A., \& Young, M. J. (1989). Bedside Case Presentations: Why Patients like Them But Learners Don’t. Journal of General Internal Medicine, 4, 284-287.

http://dx.doi.org/10.1007/BF02597397 